\title{
Retroperitoneal abscess with concomitant hepatic portal venous gas and rectal perforation: a rare triad of complications of acute appendicitis. A case report
}

\author{
Michele Diana, Alexandre Paroz, Nicolas Demartines', Markus Schäfer
}

\begin{abstract}
Background: While retroperitoneal abscess is a known complication, hepatic portal venous gas and rectal perforation have not been reported as a concomitant sequelae of acute appendicitis. Here we report a case of a patient with a perforated appendicitis that was associated with these triad of complications.

Materials and Methods: In addition to report our case, we carefully reviewed the literature in order to detect similar cases and the causes of such rare conditions.

Results: Only 26 cases (including our patient) of acute appendicitis complicated by retroperitoneal abscesses have been published in the English literature between 1955 and 2008. There was one case having hepatic portal venous gas, and one further case with a rectal perforation associated with acute appendicitis. All patients with retroperitoneal abscess presented with non specific clinic symptoms that not revealed any suspicion for such a complicated disease. Hence, delayed diagnosis and treatment are not uncommon.

Conclusions: So far, no patient has been described with such a triad of rare complications related to acute appendicitis. We want to emphasize the insidious onset of retroperitoneal abscess formation, and the need of prompt recognition and adequate treatment to avoid deleterious outcome.
\end{abstract}

\section{Introduction}

Acute appendicitis is a very common disease with low morbidity and mortality rates in most countries. While uncomplicated appendicitis can easily be treated, complicated appendicitis with perforation and abscess formation remains a challenging treatment. In particular, large abscess and advanced peritonitis often require repeated surgical interventions combined with percutaneous drainage performed by interventional radiology, as well as intensive care and antibiotic treatment. Such treatment is associated with markedly increased complications, e.g. sepsis, prolonged ileus, and adhesion formation [1]. The development of incisional hernia, recurrent bowel obstruction, and impaired fertility rates in female patients are the main adverse events during long-term

\footnotetext{
* Correspondence: demartines@chuv.ch

Department of Visceral Surgery, Centre Hospitalier Universitaire Vaudois, University Hospital, Lausanne, Switzerland
}

course [2]. In contrast, bowel necrosis with portal venous gas formation is very rare after complicated appendicitis [3]. Here we report a case of an extensive retroperitoneal abscess formation with rectal perforation and portal venous gas embolization after necrotizing acute appendicitis in a young male patient.

\section{Case report}

A 43-year old man was admitted to the Emergency Department with progressive abdominal pain, nausea, reduction in defecatory frequency and change in stool appearance as hard separate lumps that started almost three weeks before, and in addition, new onset of anal bleeding. There were no preexisting co-morbidities. The patient had tachycardia (up to $140 \mathrm{bpm}$ ), arterial hypertension $(170 / 70 \mathrm{mmHg})$ and fever $\left(38^{\circ} \mathrm{C}\right)$. Clinical examination revealed an abdominal distension with a palpable mass in the lower abdomen, as well as signs of

(c) 2010 Diana et al; licensee BioMed Central Ltd. This is an Open Access article distributed under the terms of the Creative Commons 
peritoneal irritation. The rectal examination was very painful, and an ulcerative lesion was perceived on the anterior rectal wall. Anal bleeding could be confirmed.

The laboratory findings revealed increased C-reactive protein (CRP) levels up to $100 \mathrm{mg} / \mathrm{l}$, leucocytes $8.8 \mathrm{G} / \mathrm{l}$, and serum lactate levels of $4.5 \mathrm{mmol} / \mathrm{l}$.

The abdominal CT scan with only IV contrast showed a perforation of the anterior rectal wall, $10 \mathrm{~cm}$ proximally from the anorectal border with multiple, partially confluent large abscesses located extra- and retroperitoneally (Figure 1). A significant air collection ascended from the lower pelvis through the retroperitoneal space up to the left kidney (Figure 2). Finally, massive hepatic portal venous gas was detected (Figure 3). Due to a coprolith and local abscess formation, appendiceal perforation was also highly suspected (Figure 1).

The patient underwent emergency laparotomy. Intraoperatively, a necrotizing appendicitis was found with multiple abscess formation in the retroperitoneal space. The abscess extended from the perirectal area in the pelvis up to the left kidney. The sigmoid colon, the upper and mid rectum were surrounded by the abscess. Perforation of the anterior rectal could be confirmed. Sigmoid and the upper two third of the rectum were resected, and a Hartmann's situation created. The appendix was excised and all abscess were drained by widely opening the retroperitoneal space.

Due to the severe sepsis, the patient stayed for three days in the ICU, and another 18 days on the normal ward. Initial blood cultures were positive to Bacterioides fragilis and turned sterile after a week. Cultures of the abscesses were positive to Bacterioides fragilis, Escherichia coli and Streptococcus anginosus. IV antibiotic treatment (Piperacillin-Tazobactam $4.5 \mathrm{~g}$ three times

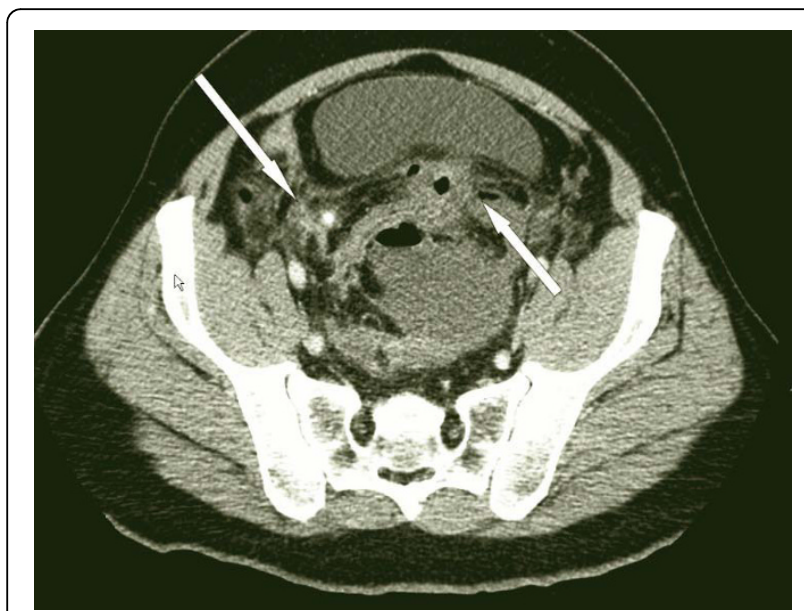

Figure 1 CT Scan showing a necrotic appendix with a stercolith (long arrow) and anterior wall perforation (short arrow).

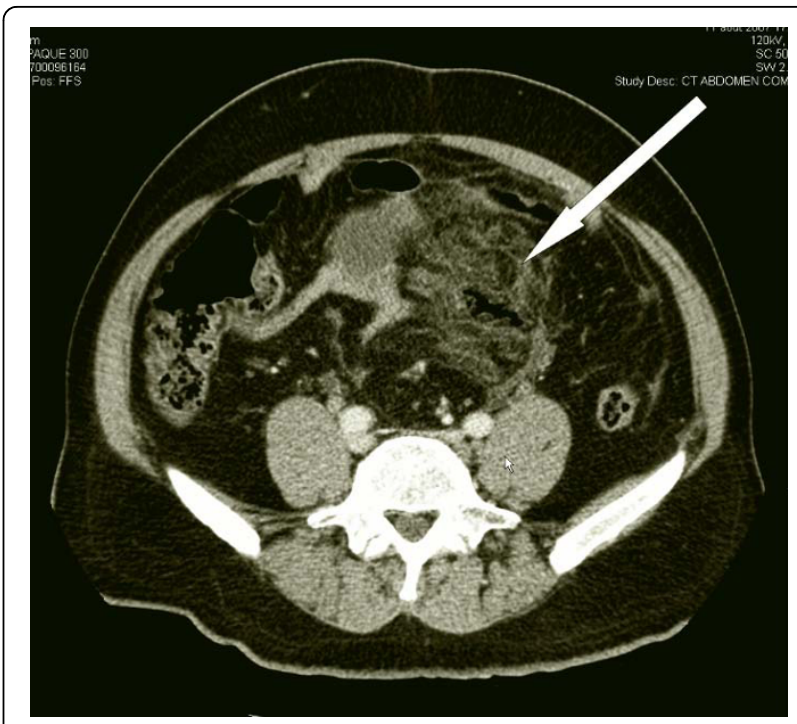

Figure 2 Retroperitoneal phlegmon with some air bubbles.

per day) was performed for 15 days and a further per os antibiotic treatment (Levofloxacin $500 \mathrm{mg}$ twice per day) was introduced for 7 days. The patient fully recovered, and was finally discharged after 21 days. Restoration of the bowel continuity was performed after 3 months. During follow-up of one year, the long-term course was uneventful.

Histopathology showed a perforated appendicitis with severe peritonitis, as well as large necrosis formation of sigmoid mesenteric adipose tissue and a necrotic ulcer measuring $1 \mathrm{~cm}$ square on the anterior wall of the rectum. Since no diverticular disease could be detected, it was strongly assumed that necrotizing appendicitis

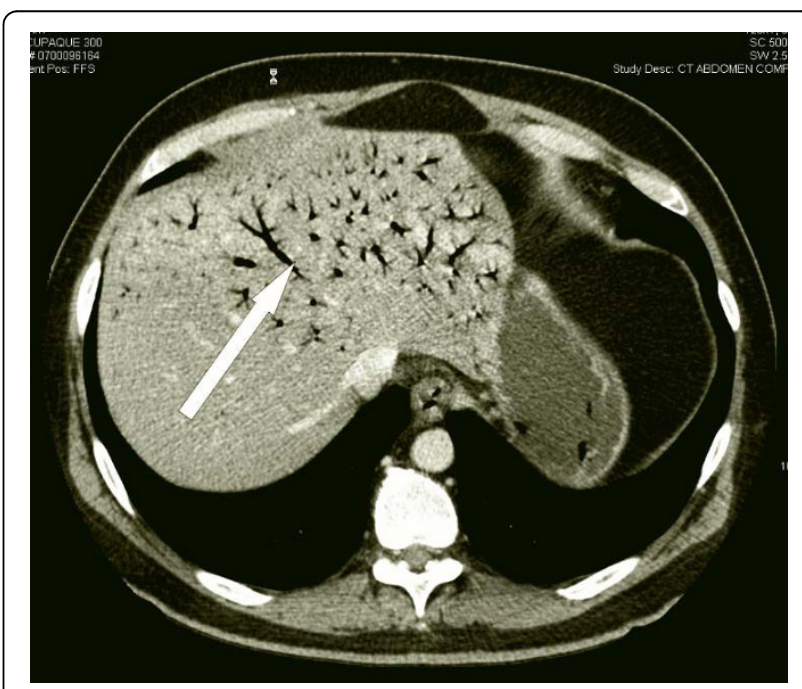

Figure 3 Hepatic portal venous gas in several intrahepatic portal branches. 
being the trigger of this massive inflammatory process that also facilitated rectal wall necrosis and stercoral perforation, respectively.

\section{Discussion and review of the literature}

\section{Retroperitoneal abscess and acute appendicitis}

Large retroperitoneal abscess represents a potentially life-threatening complication of hollow viscus organ perforation, e.g. appendicitis [4,5], diverticulitis [6], as well as inflammatory diseases of the pancreas [7] and kidneys [8]. Often its starts as a retroperitoneal phlegmon with few clinical symptoms, hence its timely diagnosis may not be always achieved. Once abscess formation has started, it may spread from the pelvis along the spine and psoas muscle up to the diaphragm and laterally to the abdominal wall since there are no anatomical barriers limiting its penetration. Perforation of the appendix into the retroperitoneal space probably represents one of the commonest reason for large retroperitoneal abscess formation but there are only few reported series in the literature [4]. While its real incidence remains unknown, several risk factors have been identified to promote large abscess formation, such as diabetes, alcohol abuse, liver cirrhosis, malignancy, chronic renal failure, and immunosuppressive therapy [9].

Hsieh et al. recently reported two cases and summarized the literature, whereby they found only additional 22 cases [4]. The main clinical features are the delayed diagnosis (mean time until diagnosis of 16 days), symptoms are dependent on the localization of the abscess and often unspecific, extension of abscess formation into the thigh and perinephritic space, and an increased disease-related mortality of $19 \%$. Similar to our case, final diagnosis of retroperitoneal perforation originating from acute perforated appendicitis is often only achieved during surgical exploration. However, it remains unclear, who an otherwise healthy young patient can develop such a major abscess without having more clinical symptoms.

\section{Hepatic portal venous gas and acute appendicitis}

The presence of air bubbles in the extrahepatic and/or intrahepatic portal venous system is primarily a radiological finding that is detected by performing an abdominal CT scan for various reasons. Despite portal venous gas is generally a late feature of advanced intestinal necrosis with an increased mortality, there are various other clinical conditions that may also cause portal venous gas formation, i.e. inflammatory bowel disease, biliary tract infections, cardiac and liver transplantation, acute pancreatitis, and blunt abdominal trauma [10]. It is assumed that gas may enter the portal venous system by an intestinal mucosal damage and increased intraluminal pressure, or gas-forming bacteria may translocate through the bowel wall during abdominal sepsis.
While bowel necrosis was the predominant reason for portal venous gas formation, non-ischemic reasons have become more frequent during recent decades [11]. Due to the latter reasons, overall morbidity decreased from $75 \%$ to $39 \%$.

Portal venous gas formation due to perforated appendicitis has been previously reported in two cases $[3,12]$. In our patient, portal venous gas formation could potentially be induced by both, perforated appendicitis and rectal perforation, respectively. However, it was assumed that rectal perforation was a secondary complication of the retroperitoneal abscess which occurred as a sequelae of perforated appendicitis.

\section{Rectal perforation and acute appendicitis}

Rectal perforation and necrosis represents an extremely rare event after retroperitoneal abscess formation. So far, only one case of rectal necrosis and simultaneous pelvic abscess as a consequence of perforated appendicitis was published in 1968 by Gostev [13]. In our patient, it remains somewhat unclear, which was the pathophysiology of rectal perforation. Ischemia, pre-existing inflammatory bowel disease, and manipulation as the commonest reasons could be excluded. Thus, impacted stool due to abscess-related impaired bowel motility caused a so-called stercoral perforation.

\section{Conclusion}

In conclusion, this patient presented with three very rare complications of acute appendicitis that all occurred at the same time. Despite the delayed diagnosis, the final outcome was good due to the rapid surgical intervention that aimed to control all infectious areas in order to assure patient's survival.

\section{Authors' contributions}

MD and AP drafted the manuscript, ND et MS critically revised the manuscript. All authors read and approved the final manuscript.

\section{Competing interests}

The authors declare that they have no competing interests.

Received: 14 October 2009

Accepted: 28 January 2010 Published: 28 January 2010

\section{References}

1. Blomqvist PG, Andersson RE, Granath F, Lambe MP, Ekbom AR: Mortality after appendectomy in Sweden, 1987-1996. Annals of surgery 2001, 233(4):455-460.

2. Tingstedt B, Johansson J, Nehez L, Andersson R: Late abdominal complaints after appendectomy-readmissions during long-term followup. Digestive surgery 2004, 21(1):23-27.

3. Tsai JA, Calissendorff B, Hanczewski R, Permert J: Hepatic portal venous gas and small bowel obstruction with no signs of intestinal gangrene after appendicectomy. The European journal of surgery = Acta chirurgica 2000, 166(10):826-827.

4. Hsieh $\mathrm{CH}$, Wang $\mathrm{YC}$, Yang $\mathrm{HR}$, et al: Retroperitoneal abscess resulting from perforated acute appendicitis: analysis of its management and outcome. Surgery today 2007, 37(9):762-767. 
5. Tomasoa NB, Ultee JM, Vrouenraets BC: Retroperitoneal abscess and extensive subcutaneous emphysema in perforated appendicitis: a case report. Acta chirurgica Belgica 2008, 108(4):457-459.

6. Busel D, Ossa G: [Retroperitoneal abscess due to perforated diverticulitis. Diagnosis and percutaneous drainage guided by ultrasonics]. Revista medica de Chile 1987, 115(6):569-570.

7. Tai SS, Foo NP, Lin HJ, Tseng JC: Severe complication of pancreatitis huge retroperitoneal abscess formation. Pancreatology 2007, 7(1):86-87.

8. Capitan Manjon C, Tejido Sanchez A, Piedra Lara JD, et al: Retroperitoneal abscesses-analysis of a series of 66 cases. Scandinavian journal of urology and nephrology 2003, 37(2):139-144.

9. Crepps JT, Welch JP, Orlando R: Management and outcome of retroperitoneal abscesses. Annals of surgery 1987, 205(3):276-281.

10. Peloponissios N, Halkic N, Pugnale M, et al: Hepatic portal gas in adults: review of the literature and presentation of a consecutive series of 11 cases. Arch Surg 2003, 138(12):1367-1370.

11. Kinoshita $H$, Shinozaki $M$, Tanimura $H$, et al: Clinical features and management of hepatic portal venous gas: four case reports and cumulative review of the literature. Arch Surg 2001, 136(12):1410-1414.

12. Lubin JS: Portomesenteric air from acute necrotizing appendicitis. Int J Emerg Med 2009, 2(2):123-124.

13. Gostev VS: [Necrosis of the rectum in a pelvic abscess of appendicular origin]. Vestnik khirurgii imeni / / 1968, 100(1):118-119.

doi:10.1186/1749-7922-5-3

Cite this article as: Diana et al.: Retroperitoneal abscess with concomitant hepatic portal venous gas and rectal perforation: a rare triad of complications of acute appendicitis. A case report. World Journal of Emergency Surgery 2010 5:3.

\section{Submit your next manuscript to BioMed Central and take full advantage of:}

- Convenient online submission

- Thorough peer review

- No space constraints or color figure charges

- Immediate publication on acceptance

- Inclusion in PubMed, CAS, Scopus and Google Scholar

- Research which is freely available for redistribution

Submit your manuscript at www.biomedcentral.com/submit
Biomed Central 